\title{
Écriture de soi et de l'autre: Récits de filiation et contestation chez Herzog et Picasso
}

\author{
Writing about Oneself and Others: Récits de \\ filiation and Objection - Herzog and Picasso
}

\author{
BÉATRICE VERNIER \\ Lakehead University \\ bvernier@lakeheadu.ca
}

\begin{abstract}
This paper examines two "récits de filiation", Un héros by Félicité Herzog, the daughter of the famous mountaineer Maurice Herzog, and Grand-père by Marina Picasso, the daughter of the renowned artist Pablo Picasso. These authors dispute the public image of these celebrities by demonstrating that it does not correspond to the egotist and cruel person that they knew during their childhood. Although the authors seem to reject their filiation with these individuals, we show their willingness to accept the filiation with a father as a great mountaineer and a grandfather as a unique painter, but not with the man corrupted by celebrity. These accusations, which modify the public images of these famous individuals, also correspond to the authors' desire to share with the public their profound pain. By expressing their suffering, the authors free themselves from resentment towards these famous figures, recognizing the context in which they lived and forgiving them. Maurice Herzog and Pablo Picasso were victims of their unconditional admirers, who idolized them as a hero or a genius, a role that these two figures continued to play with their relatives.
\end{abstract}

Key-words

Récit, filiation, inheritance, fame.

\begin{abstract}
Resumen
Este artículo analiza dos "récits de filiation", Un héros de Félicité Herzog, hija del gran alpinista Maurice Herzog, y Grand-père de Marina Picasso, nieta del artista famoso Pablo Picasso. Estas autoras refutan la imagen pública de su padre y de su abuelo, demonstrando que no representa el hombre inhumano que ellas conocieron durante su infancia. Aunque las autoras parecen rechazar la filiación con estas celebridades, demostramos su deseo de aceptar la filiación con un padre gran alpinista y con un abuelo artista innovador, pero no con el hombre corrupto por su renombre. Las acusaciones que cambian la imagen de estas celebridades corresponden también al deseo de las escritoras de compartir su dolor personal desconocido hasta ahora. Al revelar sus sufrimientos, las autoras se liberan de su resentimiento hacia estas figuras famosa, toman conciencia del contexto en que vivían y las disculpan en parte. Maurice Herzog y Pablo Picasso fueron víctimas de sus admiradores incondicionales que los elevaban al rango de héroe o de genio, papel que estas figuras famosas continuaban jugando en privado.
\end{abstract}

Palabras clave

Relato, filiación, herencia, celebridad. 


\section{Introduction}

Depuis les années 1980, une forme particulière de l'écriture de soi, qui entremêle autobiographie et biographie, occupe la scène littéraire française. L'auteur se raconte en effet de manière intime par le biais du récit sur un parent ou un ancêtre décédé et il fut difficile au départ de catégoriser ce type d'écrit. Bruno Blanckeman dans son ouvrage Les Récits indécidables leur attribua le terme "Récits transpersonnels", qu'il caractérisait comme suit: “Si le récit autofictionnel s'attache à mettre en verbe l'autre du moi, un certain type de récit autobiographique semble préoccupé par le moi en l'autre" (Blanckman, 2008: 22). Dominique Viart leur a finalement conféré en 1996 la dénomination récit de filiation ${ }^{1}$ qui répond à la définition suivante: "Cette forme littéraire a pour originalité de substituer au récit plus ou moins chronologique de soi qu'autofiction et autobiographie ont en partage, une enquête sur l'ascendance du sujet" (Viart, 2009: 96).

Le but premier de ces récits correspond à la quête d'un père ou d'un ancêtre décédé mal connu, ou avec qui l'auteur juge ne pas avoir eu une relation satisfaisante (père alcoolique, violent, ou absent par exemple). Il s'agit pour Viart "du défaut de transmission dont les écrivains présents, ou leurs narrateurs, s'éprouvent comme les victimes" (Viart, 2009: 97). Cependant, sans grandes références sur cet autre, l'auteur peine à élaborer sa biographie. Il se replie alors sur ses souvenirs d'enfant souvent disparates, sur ce dont il se souvient de cet individu ou sur des bribes de conversation captées pendant l'enfance. L'écriture autobiographique, bien que morcelée, nourrit ainsi l'entreprise biographique; tel un puzzle, elle reconstitue progressivement une image plus complète de cet autre et rétablit ainsi un lien avec lui. Viart relève par ailleurs l'importance pour l'auteur de rendre hommage à ce proche anonyme le plus souvent de condition modeste: "Cela se comprend aisément: rien ne témoigne de la vie d'un homme banal. On n'en tient pas registre, ni mémoire" (Viart, 2008: 80). Ainsi, l'écrivain J.M.G. Le Clézio souligne, dans son récit L'Africain, le travail solitaire et remarquable de son père médecin de brousse qui a soigné avec peu de moyens des populations isolées au Cameroun durant la colonisation française. Dans son ouvrage Mémé, l'acteur Philippe Torreton honore la vie de sa grand-mère, à partir de ses souvenirs d'enfant. Cette paysanne de la campagne normande, veuve, connue simplement de ses voisins et de sa famille, aurait pu être oubliée mais Torreton la magnifie et la ressuscite en insistant sur une femme qui travailla sans relâche et qui affronta la vie telle qu'elle se présentait. Laurent Demanze remarque à juste titre au sujet de ces récits: “il ne s'agit pas seulement de s'adresser aux êtres disparus, de rétablir un dialogue en dépit de la mort, mais aussi de transformer

1 Lors du colloque 'États du roman contemporain" à Calaceite, 6-13 juillet 1996. Il précisera cette notion dans l'article, Dominique Viart. 1999. "Filiations littéraires" in Etats du roman contemporain, sous la direction de Jan Baetens et Dominique Viart. Paris, Lettres modernes Minard (coll. Ecritures contemporaines), 115-139. Il reconnaîtra ultérieurement la raison de cette difficulté à qualifier ces récits: "il semblait d'abord que le souci de l'ascendance ne fut qu'un élément dans un projet plus vaste d'écriture de soi" (Viart et Vercier, 2008: 79). 
un mutisme imposé — par le statut social ou les contraintes historiques - en parole libératrice" (Lapointe et Demanze, 2009: 22). Remarquons qu'à côté de ces écrits dans lesquels les auteurs redonnent une place méritée à des individus, qui bien souvent par leur condition sociale ne purent atteindre leur plein potentiel, se profilent des récits de filiation de pères ou d'ascendants renommés dont l'objectif, nous allons le voir, diffère de ceux qui évoquent des parents anonymes.

En effet, la recherche de cet autre reconnu de la société ne peut se limiter à l'évoquer uniquement dans sa vie quotidienne personnelle puisque sa célébrité l'a conduit à évoluer également dans la sphère publique. Les enfants ou descendants, ayant bien souvent été tenus éloignés de leur notoriété signalent leur malaise à superposer l'homme public dont parlent leurs admirateurs et celui qu'ils côtoyaient dans l'intimité. Ils élaborent alors un récit qui cherche à mieux cerner le personnage médiatique afin d'obtenir un portrait plus complet de cet individu. Dans Le Bruit des clés, Anne Goscinny, fille du bédéiste René Goscinny, a du mal à rapprocher l'homme public aux héros loufoques et comiques ${ }^{2}$ dont raffolent les lecteurs, au père menant une vie ordinaire et ayant été profondément marqué par la déportation d'une partie de sa famille: "Quand on me parle de toi, on ne me parle pas de mon père, mais de René Goscinny” (Goscinny, 2012: 85). Mazarine Pingeot, fille naturelle de l'homme politique François Mitterrand, tentait dans son premier ouvrage, Bouche cousue, de mieux définir la figure paternelle en confrontant l'homme public distant et manipulateur au père respectueux et attentionné qui l'éleva. Lors d'un entretien en 2012 pour le magazine L'Express, elle reconnaît à la question de savoir si elle lit tout ce qui s'écrit dans la presse sur son père: "ce n'est pas la même personne, j'ai du mal à faire coïncider les images". Marie Nimier dans son récit de filiation, La Reine du silence, prend connaissance du brillant écrivain, journaliste et critique littéraire René Nimier, chef de file des Hussards, mais se remémore un père qui a délaissé femme et enfants. Néanmoins, la démarche scripturale que ces auteures entreprennent les aide progressivement à circonscrire une image plus précise de leur père, à se rapprocher de lui, et dès lors à renouer une filiation jusque-là fragile. Ces récits qui traitent de pères connus dans la sphère publique servent ainsi deux objectifs. A travers l'histoire remémorée de cet autre, l'auteur tente tout d'abord au niveau personnel de (re)nouer un lien avec celui dont une partie de son existence lui avait échappé. Par ailleurs, dévoiler une nouvelle image de cet homme public correspond au droit de le raconter, de se raconter, et en se réappropriant

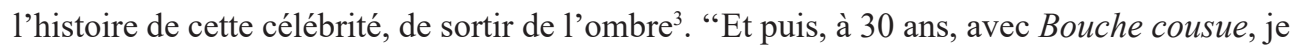
me suis autorisée à parler de cette histoire dont tout le monde parlait et qui était la mienne!", rappelle Pingeot dans le même entretien en 2012. Il est cependant important de remarquer l'intention récente de certains auteurs de dévoiler le portrait intime d'un membre de leur

2 Scénariste entre autres des personnages Astérix, Lucky Luke, et Le Petit Nicolas.

3 Voir l'article VERNIER-LAROCHETTE, Béatrice. 2009. "Bouche cousue et La Reine du silence: une réconciliation intime et publique" in Women in French Studies, vol. 17, 130-146. 
famille reconnu pour ses exploits, mais dont l'image publique ne leur fut pas étrangère; bien au contraire, ils estiment qu'elle fut très oppressante pendant leur enfance.

Cette étude se penche sur deux récits de filiation, Un héros, rédigé par Félicité Herzog ${ }^{4}$, fille de Maurice Herzog (1919-2012), qui fut le premier alpiniste à conquérir l'Annapurna et Grand-Père de Marina Picasso ${ }^{5}$, petite fille du grand peintre Pablo Picasso (1881-1973). Leur récit ne s'attache pas uniquement à mieux circonscrire une figure admirée du public mais plutôt à révéler un être à la personnalité destructrice envers ses proches en privé, et ainsi contrer l'image de l'artiste et de l'aventurier fort respectés dans la société. Ces deux auteures s'attaquent à deux grands personnages dont la renommée s'étend au-delà des frontières de la France. Il ne s'agit pas dans ce cas de découvrir la figure publique de ce père et de ce grandpère comme pour les auteures présentées précédemment, puisqu'elles estiment au contraire que leur notoriété a envahi l'espace intime de leur enfance. Picasso avoue, en étant adulte, avoir eu des appréhensions de tout ce qui lui paraissait démesuré et comprend lors d'une psychanalyse que “cette phobie venait de mon grand-père. Du volume qu'il avait occupé dans ma vie" (GP 188). Herzog souligne quant à elle: "Nous grandissions à l'ombre de cette légende héroïque qu'il s'était forgée d'une main amputée de ses premières phalanges, mais néanmoins de fer, depuis son retour de l'Himalaya" (UH 46). Si, pour Viart, ce qu'énonce la plupart des récits de filiation, c'est la perte d'un lien par rapport aux générations précédentes, chez Picasso et Herzog, c'est un excès de transmission des exploits de ces deux figures qui s'inscrit dans leur écriture. Selon elle, ces personnages ne faisaient aucune différence entre leur vie publique et leur intimité puisqu'ils exigeaient en privé l'adulation que la société leur octroyait. Toutes deux les accusent des pires exactions envers leurs proches, en particulier d'être à l'origine du suicide de leur frère respectif, Laurent et Pablito. C'est par le biais d'un regard rétrospectif sur une enfance imprégnée de la notoriété de ce personnage que chaque auteure conteste cette figure de légende en estimant que l'image adulée véhiculée dans la société ne correspondait pas à celle dénuée d'empathie qui les marqua enfant.

Néanmoins, malgré leurs accusations qui suggèrent un rejet de la filiation vis-à-vis de ces deux grandes figures, il y a, selon notre hypothèse, la volonté d'en établir une autre, celle associée au grand-père artiste, au père alpiniste, et non celle associée au peintre et à l'aventurier médiatisés. Nous montrons dans un premier temps que leurs attaques envers ces personnages célèbres sont le reflet d'une grande souffrance vécue pendant leur enfance puisque cet homme qui surpassait autrui dans son domaine (art, ascensions en haute montagne), continuait à dominer ses proches en privé. Afin de s'assurer de la validité de ce nou-

4 HERZOG, Félicité. 2012. Un Héros. Paris, Grasset. Désormais abrégé en (UH), suivi du numéro de page. Félicité Herzog, fille de Maurice Herzog est femme d'affaires, également auteure d'un second roman, Gratis publié chez Flammarion en 2015 et d'un essai "La France retrouvée” en 2017.

5 PICASSO, Marina. 2001. Grand-père. Paris, Gallimard. Désormais abrégé en (GP), suivi du numéro de page. Marina Picasso est la fille de Paulo Picasso, lui-même fils du peintre Pablo Picasso et d'Olga Khokhlova (première femme de Picasso). Héritière du $5^{\mathrm{e}}$ de la fortune du peintre, Marina Picasso s'occupe d'œuvres charitables qui viennent en aide à des enfants défavorisés au Vietnam. 
veau portrait qui contre celui d'un être fort bien considéré dans la sphère publique, elles se voient contraintes d'élaborer un récit rigoureux dont les faits peuvent être vérifiés dans les médias de l'époque ${ }^{6}$. Dans un deuxième temps, nous montrons que, révéler une souffrance personnelle réprimée depuis de longues années, les oblige à revisiter leur enfance en observatrices de cette période. Cette mise à distance, qui reconsidère la petite fille qu'elles étaient et cet individu renommé avec un regard adulte très critique, révèle tout au long du récit une tension entre l'image publique admirée et l'image privée honnie qui a imprégné et conditionné leurs jeunes années. Cette démarche scripturale, qui met en mots sans artifice les conditions dans lesquelles elles furent élevées, leur permet de prendre conscience du contexte dans lequel évoluaient ces célébrités et parviennent à résoudre cette tension. Nos deux auteures reconnaissent à la fin de leur récit qu'ils furent victimes de leur réputation et les réhabilitent, en admettant que leur comportement aurait pu être différent sans leurs admirateurs inconditionnels. Marina Picasso et Félicité Herzog renouent un lien avec ce grand-père et ce père lorsqu'elles parviennent à distinguer la problématique d'une renommée qui les emprisonna durant leur existence.

\section{Transgression d'une frontière trop longtemps respectée}

C'est de manière explicite et méthodique que chaque auteure contre l'image publique de l'artiste novateur ou de l'alpiniste hors pair en relatant leur relation à cet être peu ordinaire en privé. Dès la première phrase de leur récit, le ton est accusateur, proportionnel aux blessures affectives de leur enfance. Herzog souligne l'impact sur sa propre vie d'un père admiré pour son ascension de l'Annapurna mais dans la vie intime Don Juan invétéré peu préoccupé de ses enfants: “Toute ma vie, j’ai été dépossédée de mon père par les femmes" (UH 9). Élevée dans un milieu favorisé (famille Schneider, propriétaire des aciéries du Creusot), elle révèle une enfance dénuée d'encadrement. Son père était infidèle, et sa mère, après un divorce difficile, se retrouva seule à prendre soin de deux jeunes enfants: "Nous étions élevés comme des enfants sauvages dans un grand appartement du XVI ${ }^{\mathrm{e}}$ arrondissement nord" (UH 82). Marina Picasso débute son récit en révélant un grand-père dont la quête artistique s'effectue au dépend de ses proches jusqu'à les annihiler: "Sous le joug de sa tyrannie, mon père est né et mort de lui, trompé, déçu, avili, détruit. Inexorablement. Jouet de son sadisme et de son indifférence, mon frère Pablito s'est suicidé à l'âge de vingt-quatre ans" (GP 15); "Ma grand-mère Olga, humiliée, salie, dégradée par tant de trahisons, a terminé sa vie paralysée' (GP 16). Picasso dépeint son grand-père comme un être inaccessible, réfugié dans sa propriété dont les entrées étaient contrôlées. "Muré dans son œuvre, il avait perdu tout contact avec la réalité et s'était replié dans un monde intérieur impénétrable" (GP 193). Pour Herzog

6 Bien qu'Herzog affiche le terme "roman" sur la page titre, les faits narrés au sujet de l'ascension de son père sont identiques à ce que la presse a relaté à l'époque. Elle précise dans son entretien avec Chandellier: "Certes la matière est autobiographique mais la construction du livre est romanesque". 
et Picasso, tout est mise en scène pour le maître et l'aventurier qui ne font aucune distinction entre leurs admirateurs et leur famille puisque toute personne qui les approche se doit de les idolâtrer. "Qu'elles soient femmes, amis, enfants ou petits-enfants, ses victimes devaient être immolées à son art" (GP 132), déclare Marina Picasso. Félicité Herzog se remémore un père enfermé dans le rôle du brillant alpiniste éternel: "En société ou en famille, il jouait une représentation sartrienne du héros de l'Annapurna. Aucune contestation n'était recevable, au déjeuner avec ses propres enfants comme au cours des interviews avec les journalistes" (UH 62). Elle dénonce un père tourné vers autrui uniquement pour servir ses propres intérêts, à savoir, perpétuer l'image d'un être hors du commun:“'Autrui n'existe pas, sauf à le mythifier davantage" (UH 41).

Soulignons que les enfants d'un parent célèbre déplorent souvent l'attitude des admirateurs qui leur imposent une image élaborée par la société, ce qui les contraint en retour à s'y conformer, à dissimuler la réalité d'un vécu douloureux et donc la difficulté à affirmer leur propre identité. Pingeot remarque que le public s'attend à ce que les enfants de parents renommés partagent systématiquement leurs idées, "comme si ça coulait dans le sang" (Pingeot, 2012: 82). Félicité Herzog et Marina Picasso se souviennent d'avoir été confrontées à ce phénomène pendant leur enfance et d'avoir dû tenir le rôle de riche héritière d'un génie alors que leur existence se caractérisait, pour Picasso, par une misère matérielle et affective, et pour Herzog par un profond désarroi. "J'avais à jouer le rôle de fille du grand héros" (UH 46), se rappelle-t-elle alors qu'elle était une adolescente écrasée par la célébrité d'un père absent et méprisant envers elle et son frère. Elle rapporte ainsi les dires d'un interne lors d'une douloureuse suture du cuir chevelu sans anesthésie locale aux urgences d'un hôpital: "“Et puis quand on est la fille d'un héros pareil, on est une petite dure, non?"' (UH 171). Marina Picasso rectifie au moment de l'écriture des idées faussement circulées à l'école sur son train de vie et sur celui de son frère: "Chauffeur, garde du corps et riches alors que, ce matin, nous avons quitté la maison le ventre creux" (GP 52). Elle et son frère vivaient en effet dans une grande pauvreté avec leur mère alcoolique séparée du fils du grand peintre: "Les repas irréguliers, les tartines sans beurre trempées dans le lait chaud, les œufs brouillés à la pulpe de tomate, les pâtes sauce misère, le riz des sans-le-sou" (GP 79). Elle se remémore la situation de son père qui quémande son salaire de chauffeur à son patron qui n'est autre que son père, le grand peintre Picasso. Ce nom illustre qui s'impose dans leur vie privée, une vie quotidienne loin de la gloire de ce proche, soulève fréquemment le désir de s'en défaire à l'époque ${ }^{7}$. Pour Marina Picasso, porter le nom de son grand-père la restreignait par exemple à trouver un travail d'été ou à rendre des invitations à ses amis aisés. Herzog souffrait de l'ambiguïté de sa situation puisque son père était fort bien vu dans la société alors qu'elle et son frère étaient élevés par une mère célibataire, ce qui à l'époque et surtout dans son milieu familial aux

7 Anne Goscinny signale qu'elle a désiré après la mort de son père ne plus porter le nom de Goscinny lorsqu'elle voulait évoquer sa vie de petite fille avec un père encore vivant: "Ta célébrité même (surtout?) posthume m’interdisait ce mensonge" (Goscinny, 2012: 29). 
idées conservatrices, était peu courant: "nous étions des spécimens, pratiquement les seuls enfants de parents divorcés de nos écoles" ( $U H$ 88). Cette frontière privée/publique qu'elles ont respectée jusque-là, c'est-à-dire de n'avoir jamais révélé une enfance en décalage total avec l'existence de leur père et de leur grand-père, elles la transgressent par cet écrit. Elles insistent sur le contraste de l'enfant solitaire et désemparée qu'elles étaient, face à un être puissant, car admiré pour ses exploits. L'écriture actuelle contrebalance cette abnégation de soi sur laquelle elles se sont construites, en élaborant un portrait impitoyable de cet autre.

Ces deux figures confinées dans un monde lié à leurs prouesses, contraintes de plaire à ce public qui les encense et qui contribue à leur célébrité, ont développé une séduction sans limites. Nos deux auteures les réduisent à des êtres conduits par des pulsions primaires, surtout envers les femmes qu'ils charment et anéantissent. Herzog mentionne "le séducteur mutilé par l'exploit, le cannibale du sexe" ( $U H 40)$, et rappelle un être capable d'annihiler autrui, de l'engloutir selon ses envies: "Sa voix, son effluve, pourtant attirants et puissamment masculins, suscitaient chez moi une méfiance extrême [...]. Il était un ogre. Mon ogre de père" (UH 23). Pour Marina Picasso, "Soumises à sa sexualité animale, il les domptait [les femmes], les envoûtait, les aspirait, les écrasait sur sa toile. Lorsque des nuits durant il en avait tiré la quintessence, il les rejetait exsangues" (GP 194). Ces deux êtres encensés par le public puisqu'ils avaient pu dépasser les limites du possible (l'un imposer une nouvelle esthétique picturale, l'autre vaincre l'un des plus hauts sommets du monde), nos auteures estiment nécessaire de révéler les implications de leurs agissements trop longtemps limitées à la sphère privée. Il est important de souligner que, pour Georges Gusdorf, corriger des méprises est une des motivations principales du récit de soi:

Je veux savoir qui je suis; je ne suis pas ce que j'ai l'air d'être, aux yeux des autres comme à mes propres yeux; j'ai vécu jusqu'ici selon l'ordre des illusions familières, familiales et sociales. Je ne peux me contenter de vivre à la périphérie de moi-même, j'ai un secret, je suis un secret pour moi-même, et je suis seul à pouvoir le déchiffrer. Le commencement des écritures du moi correspond toujours à une crise de la personnalité; l'identité personnelle est mise en question, elle fait question; le sujet découvre qu'il vivait dans le malentendu Le repli dans le domaine de l'intimité répond à la rupture d'un contrat social fixant le signalement d'un individu selon l'ordre d'apparences usuelles dont l'intéressé s'aperçoit brusquement qu'elles sont abusives et fondées (Gusdorf, 1991: 23).

L'écriture ici se doit de rétablir la vérité, c'est-à-dire de modifier cette image publique dont nos deux auteures estiment avoir été victimes en ayant recours à une écriture rigoureuse, adressée à un lectorat, car comme Jean-Philippe Miraux le note: "si la question de l'exactitude préoccupe tant l'écrivain, c'est que se pose à lui, d'une façon incessante, la problématique épineuse du destinataire" (Miraux, 2005: 8).

Si ces deux écrits ont pour motivation première d'offrir au public une image plus complète de ces deux célébrités, ils autorisent aussi Picasso et Herzog à se libérer de souf- 
frances antérieures et par la même à se démarquer de cette image publique dont elles étaient prisonnières jusque-là. Afin de s'assurer de la crédibilité de leurs propos, elles valident leurs accusations en rappelant des faits que les lecteurs peuvent vérifier.

\section{Crédibilité des affirmations: preuves médiatiques et porte-parole des tiers}

Notons que l'écriture véridique à laquelle les auteurs tiennent dans les récits de filiation provoque souvent une gêne à la parution de l'ouvrage puisqu'ils dévoilent l'intimité d'un individu sans son accord. Lors d'une interview, Le Clézio souligne à la parution de son livre L'Africain sur son père médecin: "Tout ce que je raconte dans ce livre, il [mon père] l'aurait d'abord nié, et vraisemblablement peu apprécié". Dans le cas d'un individu médiatisé, les révélations péjoratives sur sa vie personnelle risquent d'avoir des retombées plus prononcées: le danger de détruire une légende qui a contribué au renom d'un pays comme dans le cas de nos deux auteures. Herzog reconnaît, lors d'un entretien en 2012 pour le journal Le Dauphiné Libéré, avoir toujours su l'importance de l'exploit de son père pour une génération de Français, et d'avoir ainsi attendu pour s'exprimer: "Je respectais ce que ce mythe revêtait de sacralité". En effet, ici l'artiste novateur et l'alpiniste, ayant accompli une ascension hors du commun, contribuaient au rayonnement international de la France.

Ainsi, discréditer ces personnages repose sur la crédibilité des affirmations, ce à quoi nos deux auteures sont sensibles. Elles vont devoir s'assurer de l'adhésion du lecteur à venir. Thomas Clerc relève chez Leiris dans son texte, L'Afrique fantôme, de nombreuses tournures métalinguistiques qu'il attribue au désir de légitimer son discours (Clerc 2001: 91) ${ }^{8}$. Picasso, préoccupée par la réception de son récit, justifie sa position dès le début de son entreprise. Elle souligne qu'elle ne désire en aucun cas attaquer la renommée de son grand-père mais simplement témoigner d'une réalité vécue, la sienne et celle des membres de sa famille:

Mon propos n'est pas de dire du mal de Picasso. Mon propos est de tenter d'expliquer le long chemin de croix qu'il m'a fallu gravir pour tenter de réhabiliter l'image d'un homme incapable d'aimer. Mon propos est de rendre palpable la souffrance des victimes (GP 35).

A nouveau, à la fin de son récit, elle anticipe les réactions de lecteurs potentiels et leur répond comme faisant face, selon Gisèle Mathieu-Castellani, à un "tribunal imaginaire" (Mathieu-Castellani, 1996: 39): “Je sais ce que certains vont dire: - Son grand-père est célèbre, il lui laisse une fortune, elle a beaucoup d'argent ... Mais de quoi se plaint-elle? Je ne me plains pas. Je ne fais qu'entrouvrir ma mémoire et raconter les faits tels que je les ai vécus" (GP 185). Afin de valider ses affirmations, elle s'appuie sur des exemples marquants

8 Il s'agit d'un journal où, en tant que secrétaire, Leiris rapporte la progression d'une mission ethnographique en Afrique. 
de la vie des membres de sa famille parus dans les médias. Son frère Pablito meurt le jour de l'enterrement de leur grand-père, leur père deux ans plus tard, les compagnes et épouses successives, Marie-Thérèse Walter et Jacqueline Laroque s'enlèveront la vie, enfin Dora Maar mourra dans la misère au milieu des toiles de Picasso (GP 198). Herzog base certaines de ses accusations sur des articles de journaux précis et des photos publiées dans des magazines de l'époque afin de mieux étayer l'argument d'un père fabulateur et manipulateur lorsqu'elle évoque sa conquête de l'Annapurna qui, selon elle, révèle des incohérences. Elle qualifie cette ascension de supercherie puisqu'elle suggère lors de son entretien pour Le Dauphiné Libéré qu'elle se serait terminée avant le sommet, "afin d'échapper à une mort certaine":

Une photo de mon père, plus tard mondialement diffusée, est prise de mon père, debout sur l'arête en devers, sous une corniche, alors que le cliché pris de Louis Lachenal montre ce dernier, assis, sombre, jetant à peine un regard à l'objectif. Personne ne s'intéressera jamais à cette dernière image (UH 58).

Ces accusations, fort bien documentées, participent à élaborer pour le public un portrait qui diffère de ce qu'il connaissait de ces deux célébrités. Il s'agit aussi pour les deux auteures de redéfinir leur propre image publique, loin des héritières fortunées qu'on leur attribuait, ainsi que le remarque amèrement Marina Picasso: "Nous étions des Picasso, des mortnés pris au piège de la spirale d'espoirs bafoués" (GP 15). Quant à la large place qu'elles accordent dans leur récit à l'existence de leur frère respectif qui s'enlèvera la vie, il y a la volonté, certes, d'exprimer la souffrance d'un être qui leur était cher, mais nous montrons qu'il s'agit également d'une stratégie supplémentaire pour mieux convaincre autrui du pouvoir destructeur de ces deux icônes.

\section{La voix du frère disparu}

Remettre en question ce père et ce grand-père admirés en public mais craints en privé ne peut s'effectuer sans évoquer leur frère dont elles étaient proches en âge, deux ans pour Herzog et dix-huit mois pour Picasso. Pour chacune d'elles, parler de soi, c'est parler immanquablement de celui auquel elles étaient très attachées et dont elles ont tout partagé enfant. Picasso se remémore une fusion indestructible avec ce frère, ce qui transparaît par les expressions "Pablo et moi" et le "nous" qui dominent son récit:

Nous faisons les mêmes rêves, rions au même moment, avons les mêmes élans, les mêmes attitudes, vivons les mêmes mystères. Nous sommes copies conforme. Nous ne pouvons pas vivre l'un sans l'autre. Nous sommes des siamois. Rien ne nous séparera $(G P, 111)$.

Bien qu'Herzog insiste sur une enfance mouvementée aux côtés de son frère, puisqu'elle était victime d'actes de violence de sa part, elle dévoile leur attachement mutuel 
dans une vie où l'encadrement adulte était inexistant. Aimer ce frère, c'était amoindrir sa propre solitude et surtout s'assurer d'un soutien affectif dans une existence vide de sens: "Il fallait qu'il reste un tyran debout: que serais-je devenue s'il tombait?" (UH 100). Il s'agit d'évoquer celui dont la destinée fut le contraire de ce père et de ce grand-père. Ce frère représente le pendant de cette figure renommée qui l'a tant écrasé puisqu'il fut négligé, ne connut qu'une existence brève et n'eut aucune reconnaissance publique De plus, son suicide, qui était un geste fort décrié à l'époque, fut marqué du sceau du silence. L'écrit d'Herzog et de Picasso s'associe à une plaidoirie qui s'attache à défendre ce frère qui les aida à sa façon à survivre à une enfance chaotique et qui, selon elles, fut la grande victime du peintre et de l'alpiniste exceptionnels.

Picasso démarre son écrit par le suicide de son frère et reprend cette scène plus en détails lorsqu'elle le clôt. Encadrer son récit par sa disparition, c'est en révéler l'importance pour elle. Brisée par son décès, cette fusion qu'elle connue avec cet autre dans l'enfance ne cesse de l'habiter, et c'est naturellement qu'elle en vient à le défendre en revenant sur son geste imparable: “C'est moi qui l'ai trouvé baignant dans son sang, œsophage et larynx brûlés, estomac détruit et cœur à la dérive” (GP 15). Elle découvre en effet Pablito après une ingestion d'eau de javel, quatre jours après la mort de leur grand-père, et précise qu'elle veillera son frère pendant trois mois pendant lesquels il fut aux prises à d'atroces souffrances. Afin de rendre son plaidoyer plus convaincant, elle rapporte ici la déclaration qu'il lui fit juste avant de mourir afin d'expliquer sa décision d'en finir:

L'empire de Picasso t'a fermé toutes les portes. Il fallait que ça cesse. Alors, tu sais quoi, Marina? ... J'ai fait ma dernière fugue. Pour te sauver, $\mathrm{j}$ 'ai fait ma dernière fugue. C'est ce que je devais faire. Un geste à leur mesure.

- Je t'en prie, Pablito!

- J'ai voulu imploser, détruire de l'intérieur toute notre souffrance. A présent, ils réaliseront que tu existes. Désormais, ils s'occuperont de toi. Tout au moins pour l'opinion publique (GP 163).

Le discours direct de son frère qu'elle insère dans son récit, crée une mise en scène qui ne peut qu'agir en leur faveur. Il s'agit de relayer un appel au secours au sujet de leur enfance négligée, qui n'avait jamais été entendu et appuyer les accusations envers un peintre illustre dont l'attitude irrévérencieuse, indifférente aux besoins de ses proches explique le geste de son frère. Elle lui donne par cet écrit la possibilité de s'exprimer puisqu'il avait déjà tenté de dénoncer en vain les agissements de cet artiste et de ceux qui le soutenaient dans son entreprise artistique. Par ailleurs, rapporter ces propos sert à valider la thèse de son récit qui est d'établir un portrait contraire à celui du grand-père célèbre. Cette volonté rejoint l'écriture d'Herzog lorsqu'elle relate l'attitude de son père vis-à-vis de son frère Laurent.

Cette dernière narre son existence en retraçant la progression d'une schizophrénie qu'elle attribue clairement, lors de l'entretien avec Chandellier, à l'attitude de leur père qui 
maintenait la légende d'un exploit en partie faux: "Ne pas être dans la vérité a pu constituer, à mes yeux, un facteur déclenchant des troubles de Laurent". Elle décrit un jeune homme dénué de convictions personnelles car conditionné depuis son plus jeune âge à honorer et à poursuivre les exploits paternels: "Mon père distillait des attentes démesurées. La vie de son fils aîné serait à l'image de celui qui avait conquis l'Annapurna: une mythologie" (UH 74). Lorsqu'elle décrit son affection, elle effectue des parallèles avec le comportement de leur père et suggère que ce dernier aurait été atteint de problèmes psychiatriques qu'il aurait transmis à son fils:

La mégalomanie du fils renvoyait à la mégalomanie du père, qui n'hésitait pas à rapporter dans un de ses livres: 'D'égal à égal, je dialoguais avec les 8000 , les géants qui m'entouraient'. Il y avait entre mon père et mon frère, dans cette inconscience, un écho: l'ignorance des réalités, d'eux-mêmes et des autres ( $U H$ 215).

Elle superpose l'apparence physique de son frère modifiée par les médicaments à celle du père amputé de l'extrémité de ses membres après son ascension mémorable: "Des mains devenues incapables, comme celles de mon père" (UH 285). Elle accuse par ailleurs l'attitude des membres de sa famille dont le milieu privilégié, obsédé par la réussite, feignait de reconnaître son affection psychiatrique et donc sa prise en charge précoce. "Il était intolérable à notre univers, dans lequel tout ne devait être que réussite, puissance, filiation superbe, séduction et légende, d'avoir un malade, mental de surcroît" (234). Ainsi, en précisant les circonstances de leurs jeunes années où Picasso et Herzog estiment avoir été méprisés par un père et un grand-père auréolés de gloire, elles légitiment la décision de leur frère de s'être supprimé. A l'aune de son récit, Herzog réattribue d'ailleurs la notion d'héroïsme, qui a tant marqué son enfance, à son frère mais qui, au contraire de son père, n'aura jamais été reconnu du public: "Je pense à mon frère, ce héros au sourire si doux, ce blessé de l'esprit livrant cette dernière bataille malgré lui” ( $U H$ 301).

Nos deux auteures, en prenant en charge le récit de ce frère disparu le réhabilitent en montrant que la motivation réelle de son geste était en grande partie liée à la personnalité de ce père et de ce grand-père, et non uniquement, à un acte de faiblesse ou à une maladie mentale. Pour elles, leur frère n'a simplement pas pu réussir à se dégager de l'emprise d'un homme qui se définissait par ses exploits plutôt que par le devoir de guider un fils et un petit-fils dans leur vie à venir.

C'est à la fois par le truchement d'accusations franches envers leur grand-père et leur père, puis en défendant le geste de leur frère, que Picasso et Herzog déconstruisent méticuleusement l'image de ces deux êtres tant admirés en société. Les mots, qu'elles parviennent à mettre sur ce qui ne fut jamais dit, leur procurent néanmoins la possibilité de reconsidérer ces deux figures renommées sous un nouvel angle. 


\section{Déplacement des accusations: une réhabilitation du personnage renommé}

Le regard rétrospectif sur ce personnage célèbre, évolue ainsi au cours de l'entreprise scripturale. Comme dans tout récit qui évoque l'enfance, le regard adulte de l'auteur recouvre progressivement celui de l'enfant d'antan qui ne pouvait rationaliser les faits et gestes de leur entourage, ce que Laurent Demanze énonce comme suit:

Le récit d'enfance rejoue ainsi un drame épistémologique, qui mène de l'énigme au savoir, de la participation sensorielle à la prise de conscience. Car la césure qui traverse l'intimité de l'individu et scinde la parole du narrateur, entre qualification du percept et nomination retardée, tient à la division ontologique de l'homme (Demanze, 2008: 199).

L'objectif principal de nos deux auteures au début de leur démarche scripturale n'était pas de partir à la découverte de ce proche célèbre, mais plutôt de révéler sa personnalité. L'écriture leur a permis néanmoins d'identifier plus précisément les tenants et les aboutissants de sa célébrité et d'en saisir les rouages, c'est-à-dire une réalité contraignante, celle de devoir jouer en tout temps un personnage de héros ou de "génie" (GP 191). Les récits de Picasso et d'Herzog s'articulent ainsi autant sur des accusations au niveau de la sphère intime - l'impact néfaste de ce personnage sur l'enfant qu'elles étaient et sur leurs proches -, que de la sphère publique - condamner l'attitude des admirateurs face à leurs exploits, comme nous allons le voir.

En se libérant de leurs souffrances d'enfant, et en réhabilitant leur frère à l'écrit, Herzog et Picasso, dégagées de tout affect, parviennent à considérer ce proche de manière plus objective. Elles repositionnent ce père et ce grand-père dans le contexte de notoriété qui les auréolait et qui, estiment-elles les maintenait dans un monde restreint à leur art d'avant-garde ou à leurs exploits alpins. "Une chose comptait: la peinture et rien d'autre" (GP 94), s'insurge Picasso. Ce confinement, Herzog l'énonce de la façon suivante: "Tout était bon pour parfaire la statue de héros qu'on lui avait demandé d'ériger autour de sa personne" (UH 41). Elle comprend que son père a été trompé par une France d'après-guerre, déjà humiliée par l'Occupation allemande, décidée également à prendre sa revanche suite à l'échec d'une expédition française sur le Karakoram en 1936 et de plus, bien décidée à "se lancer sur ce terrain de chasse britannique et allemand" (UH 50). Pour elle, cette expédition n'était en rien un exploit humain; quant à l'Annapurna “c'était un sommet politique, pas un sommet de montagne. C'était une bataille qui avait été gagnée par la France” (UH 72). Par ailleurs, toutes deux reportent leurs accusations sur ceux qui, selon elles, leur auraient fait perdre la notion d'altérité, c'est-à-dire, tous ceux dans la société qui les admiraient de façon inconditionnelle. “Pour sauver les apparences d'une ascension de légende, il a réécrit l'histoire, trahi et négligé son entourage sans jamais avoir le sentiment d'avoir fait mal puisque la société le jugeait si bien" (UH 41), dénonce Herzog au sujet des exactions de son père. Selon Picasso, son 
grand-père asservissait son entourage mais c'était avec l'aval de "tous ceux qui accordaient du pouvoir à mon grand-père, ceux qui le glorifiaient, l'auréolaient, l'élevaient à l'échelon de dieu" (GP 36). Sans excuser les méfaits de ce proche renommé, nos deux auteures, par le biais d'un récit marqué d'une souffrance personnelle notable, réussissent malgré tout à considérer ces deux célébrités de manière apaisée.

Herzog et Picasso les réévaluent de manière plus objective et arrivent à les apprivoiser, en considérant leur notoriété comme responsable de leur attitude destructrice dans leur vie privée. En filigrane d'un récit accusateur, Herzog se questionne sur l'éventualité d'un être ordinaire dont l'ascension d'un des plus hauts sommets du monde et les amputations qu'il dut subir suite à cette aventure, lui aurait fait perdre son humanité: "Peut-être les blessures, les épreuves physiques, la renommée mondiale de son exploit, les très nombreuses marques de reconnaissance dont il jouit au cours de son existence le rendaient-ils impropre au rôle de père" (UH 12). Elle semble deviner à plusieurs reprises un père sensible derrière le héros imbu de lui-même; un sentiment d'admiration se profile parfois au sein du récit mais ces impressions s'inscrivent sous la forme de questions laissées sans réponse: "Ressentait-il une secrète culpabilité à avoir entraîné Louis Lachenal ${ }^{9}$ dans cette folie, même au nom de l'intérêt national?" (UH 60); “D'où tenait-il cette extraordinaire volonté de se relever et de survivre?" (UH 63). Ces interrogations, Lori Saint-Martin les considère nécessaires dans son étude sur la représentation du père dans les romans québécois lorsque les auteurs peinent à saisir cette figure souvent absente ou silencieuse: "patiente interrogation des images du passé, [1'] ardent désir de faire la part des choses" (Saint-Martin, 2010: 148). Pour Herzog, ces questions contribuent à lui faire saisir la problématique d'un homme qui a côtoyé la mort et l'impact notoire sur sa façon d'envisager son rapport à autrui et à la vie: "Le drame de mon père est l'incommunicabilité de son expérience" ( $U H 40)$. Nous voyons ainsi que ce récit dénué de complaisance, autorise cependant nos auteures à énoncer progressivement, parfois timidement, une image nuancée de cet autre.

Ce revirement, caractéristique des récits de filiation, reflète le travail d'investigation rigoureux qui s'attache à revisiter l'individu incompris qui a marqué l'enfance pour faire surgir un être dont le portrait s'avère dénué des invectives du début du récit. Il devient alors plus aisé de voir l'être humain dissimulé derrière le génie et le héros, et par là-même de renouer un lien, une filiation à cet autre ${ }^{10}$. Pour Carine Trévisan: "Souvent voués à combler les blancs de l'histoire familiale, à rémunérer le défaut du silence des ascendants, les écrits effectuent un travail de reliaison" (Trévisan et Pinçonnat, 2011: 9). Se relier à ce personnage renommé s'effectue ici lorsque les deux auteures prennent conscience que l'image de ce

9 Louis Lachenal (1921-1955), alpiniste chevronné, fut un de ses compagnons de cordée qui atteignit le sommet de l'Annapurna avec Maurice Herzog et qui subit une amputation des pieds suite à cette expédition.

10 Par exemple, Franz-Olivier Giesbert, dans le récit sur son père, l'accuse dès le début d'avoir été un père peu attentionné et violent envers ses proches: “Mon père m'a volé mon enfance C'est à cause de lui que j'ai toujours regardé le monde avec des yeux d'adulte”, GIESBERT, Franz-Olivier. 2004. L'Américain. Gallimard, 2004. 
parent n'était autre que celle captée et conservée par l'enfant qu'elles étaient. Picasso reconnaît qu'il s'agissait d'une image délimitée à la sphère intime, et de ce que les adultes de leur entourage projetaient de cet être peu ordinaire:

À travers le prisme de mon père, il était méprisant et avare. À travers celui de ma mère, il était pervers et insensible. Jacqueline, avec ses Monseigneur, nous assenait le coup de grâce. Elle nous le présentait comme l'un de ces dieux cruels que les Aztèques célébraient par des sacrifices humains. Nourrie de cette légende, je l'ai longtemps tenu pour seul responsable de notre détresse. Tout était sa faute: la déchéance de mon père, les excès de ma mère, le déclin de ma grand-mère Olga, la dépression et la mort de mon frère Pablito. Je lui en voulais de ne s'être jamais penché sur notre sort et de nous laisser à l'abandon (GP 192).

Nos deux auteures reconsidèrent d'ailleurs au cours de l'écrit l'attitude des membres de leur famille qu'elles plaçaient au début de leur récit en victimes de ce père et de ce grand-père. Elles s'autorisent au cours de leur démarche à rendre ces proches responsables de ne pas les avoir protégées ainsi que leur frère du grand maître et de l'alpiniste hors pair: "l'irresponsabilité d'un père, d'une mère mais aussi d'une épouse possessive nous avait dépouillés d'une affection que Pablito et moi guettions à chaque visite" (GP 193), remarque Picasso. Herzog se remémore une mère seule, dépitée d'avoir été trompée par son mari, dont la vie ne correspondait pas aux attentes de sa famille et qui peinait à remplir son rôle de mère: "Ma mère, perdue dans l'écriture d'une thèse de philosophie et dans des aventures amoureuses aussi nombreuses que sans lendemain, ne nous voyait pas" ( $U H$ 111). En progressant dans leur réflexion scripturale, nos deux auteures, se dégagent de leurs blessures, de leur incompréhension d'enfant, et sont à même de se repositionner et de repositionner tous les acteurs de cette histoire douloureuse.

Le père et le grand-père du début du récit qui maltraitait leur entourage, sont reconnus dès lors victimes du monde médiatique qui les retenait dans ses rets, comme Picasso l'affirme: "mon grand-père nous a été volé" (193). Nos deux auteures peuvent alors se réconcilier avec l'être dégagé de sa célébrité car, comme Viart le souligne: "Si le texte sur l'ascendance est toujours potentiellement un récit, c'est qu'il interroge une continuité, s'emploie à rétablir un continuum familial. À restituer une expérience dont je suis le produit" (Viart et Vercier, 2008: 88). Picasso reconnaît d'ailleurs à la fin de son récit avoir accepté l'héritage de Picasso lorsqu'elle narre l'adoption d'un de ses enfants au Vietnam : "Il a trois mois à peine mais son regard est vif à travers ses paupières bridées. - Les yeux de Picasso, me glisse Mme Hôa. Je souris. Les yeux de Picasso: un héritage qui me comble de joie" (GP 203).

\section{Conclusion}

Dans les récits de filiation de personnages renommés, les descendants rétablissent une relation lorsqu'ils arrivent à cerner clairement l'homme public et à l'associer à celui qu'ils 
connaissaient en privé. Dans le cas de Picasso et d'Herzog, le travail de filiation s'effectue en dénonçant dans un premier temps ce père et ce grand-père des pires exactions, en exorcisant une souffrance perçue comme provoquée par cet autre. Elles offrent sans concession un portrait intime qui conteste une image adulée en public qu'elles estiment réductrice, en invitant le lecteur à la reconsidérer de leur point de vue. Dans un deuxième temps, comme nous venons de le démontrer, loin de se désolidariser complètement de cette figure célèbre, elles reconnaissent leur filiation à ce père et à ce grand-père lorsqu'elles comprennent l'impact d'une reconnaissance publique sur leurs agissements personnels. Elles parviennent alors à innocenter l'être qui s'était laissé corrompre par la célébrité: le grand artiste imbu de ses créations et l'aventurier fabulateur. Par le truchement de leur entreprise scripturale, Marina Picasso et Félicité Herzog refusent un lien quelconque avec l'homme public corrompu par sa renommée. En revanche, elles se sentent solidaires de l'homme victime de sa célébrité et reconnaissent leur filiation au grand-père peintre novateur et au père aventurier d'exception.

\section{Références bibliographiques}

Blanckeman, Bruno. 2008. Les Récits indécidables: Jean Echenoz, Hervé Guibert, Pascal Quignard. Villeneuve d'Ascq, PU du Septentrion.

Clerc, Thomas. 2001. Les Ecrits personnels. Paris, Hachette.

Demanze, Laurent. 2008. Ecritures orphelines. Pierre Bergougnioux, Gérard Macé, Pierre Michon. Paris, José Corti.

Demanze, Laurent. 2009. "Les possédés et les dépossédés" in Etudes françaises, vol. 45, no 3, 11-23.

Doubrovski, Serge. 2001. Fils. Paris, Gallimard, coll. "Folio".

Giesbert, Franz-Olivier. 2004. L'Américain. Paris, Gallimard.

Goscinny, Anne. 2012. Le Bruit des clés. Paris, Editions NiL.

GuSDorf, Georges. 1991. Les Ecritures du moi. Paris, Odile Jacob.

Herzog, Félicité. 2012a. Un héros. Paris, Grasset.

Herzog, Félicité. 2012b. “On ne sait pas ce qui s'est passé à l'Annapurna”, interview avec Antoine Chandellier, Le Dauphiné libéré 3 août 2012 [consulté le 15/01/2018] <https://www. ledauphine.com/isere-sud/2012/08/02/on-ne-saura-jamais-ce-qui-s-est-passe-a-l-annapurna $>$.

Lapointe, Marie-Emanuelle et Demanze, Laurent. 2009. "Figures de l'héritier dans le roman contemporain" in Etudes françaises, vol. 45, $\mathrm{n}^{\circ} 3$.

Le Clézio, J.M.G. 2004a. L’Africain. Paris, Mercure de France. 


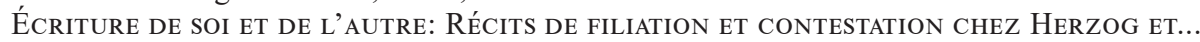

Le Clézio, J.M.G. 2004b. “J.M.G. Le Clézio: 'Mon père l'Africain””, interview avec Gérard de CORTANZE in Le Magazine Littéraire, $\mathrm{n}^{\circ}$ 430, 69.

Matthieu-Castellani, Gisèle. 1996. La Scène judiciaire de l'autobiographie. Paris, PUF. Miraux. Jean-Philippe. 2005. L'autobiographie. Ecriture de soi et sincérité. Paris, Armand Colin.

Picasso, Marina. 2001. Grand-père. Paris, Denoël,

PingeOt, Mazarine. 2012a. Bon petit soldat. Paris, Julliard,

Pingeot, Mazarine. 2012b. “Une révolte adolescente aurait été un coup d'Etat", interview avec Marianne Payot, L'Express publié le 16/10/2012; [consulté le 20/08/ 2018] <https:// www.lexpress.fr/culture/livre/mazarine-pingeot-une-revolte-adolescente-aurait-ete-uncoup-d-etat_1175171.htmlè>.

Pingeot, Mazarine. 2012. Bouche cousue. 2005. Paris, Julliard,

Saint-Martin, Lori. 2010. Au-delà du nom. La question du père dans la littérature québécoise actuelle. Montréal, PU Montréal.

Torreton, Philippe. 2014. Mémé. Paris, L’Iconoclaste.

Trévisan, Carine. 2011. "Préface" in Transmissions et filiations, sous la direction de Carine Trévisanet Crystel Pinçonnat. Revue des Sciences Humaines, n 301, 9.

Vernier-Larochette, Béatrice. 2009. "Bouche cousue et La Reine du silence: une réconciliation intime et publique". Women in French Studies, n 17, 130-146.

VIART, Dominique. 1999. "Filiations littéraires" in Etats du roman contemporain, sous la direction de Jan Baetens et Dominique Viart. Paris, Lettres modernes Minard, coll. "Ecritures contemporaines", 115-139.

VIART, Dominique. 2009. "Le silence des pères au principe du 'récit de filiation"” in Etudes françaises, vol. 45, $\mathrm{n}^{\circ}$ 3, 95-112.

Viart, Dominique et Vercier, Bruno. 2008. La Littérature Française au présent. Héritage, modernité, mutations. Paris, Bordas. 